\title{
Calibration Approach Based Estimators of Finite Population Mean in Two - Stage Stratified Random Sampling
}

\author{
Sandeep Kumar* \\ Department of Agricultural Statistics, Narendra Deva University of Agriculture and \\ Technology, Kumarganj, Faizabad-224229 (UP), India \\ *Corresponding author
}

\begin{abstract}
A B S T R A C T
\section{Keywords}

Finite population, Auxiliary information, Two-stage stratified random sampling, Calibration estimators, Population mean

Article Info

Accepted:

14 December 2017

Available Online:

10 January 2018

Deville and Sarndal (1992) developed calibration estimator by using the auxiliary information to obtain a better estimate of the population total of study variate $y$ and auxiliary variable $x$. While calibration approach does not assume any explicit relationship between $y$ and $x$ but it assume only that $X$, the population total of $x$ is known following Deville and Sarndal (1992) calibration approach, calibration estimators of finite population mean in two-stage stratified random sampling have been developed. The variances and unbiased estimator of their variances have been derived. In the present paper, an attempt has been made to conduct a limited simulation study to examine the relative performance of calibration approach based estimators. The real data has been taken from Appendix-C of Sarndal et al., (2003). The study variate y and the auxiliary variate $\mathrm{x}$ are the population of the Sweden in year 1985 and year 1975 which is divided in 284 municipalities (MU284). The results have been found that the calibration estimators have outperformed the usual estimator of finite population mean in two-stage stratified random sampling.
\end{abstract}

\section{Introduction}

The auxiliary information has been effectively used in sample surveys at selection, stratification and estimation stage for bringing about the improvement in the estimate of population parameters.

The different fundamental approaches are used in finite population survey sampling. These are design based approaches, model based approach and model assisted approach. Under design based approach, the most common unbiased estimator of finite population total $Y$ of study variable $y$ is the well-known Horvitz-Thompson estimator is given by

$\hat{Y}_{H T}=\sum_{i \in s} d_{i} y_{i}$

With variance

$V\left(\hat{Y}_{H T}\right)=-\frac{1}{2} \sum_{i=1}^{N} \sum_{j=1}^{N} D_{i j}\left(y_{i}-y_{j}\right)^{2}$ 
Where $D_{i j}=\left(\pi_{i j}-\pi_{i} \pi_{j}\right), d_{i}=1 / \pi_{i}, \pi_{i}$ being the inclusion of probability of $i^{\text {th }}$ unit in the sample $s$ which has been drawn from the finite population of size $N$ by a probability sampling design $P(\cdot)$ and $y_{i}$ is the observed value of $y$ corresponding to the $\mathrm{i}^{\text {th }}$ unit selected in sample $s$.

Deville and Sarndal (1992) developed calibration estimator of finite population total is given by

$$
\begin{aligned}
& \hat{\mathrm{Y}}_{C}=\hat{\mathrm{Y}}_{\mathrm{HT}}+\hat{\beta}\left(\mathrm{X}-\hat{\mathrm{X}}_{\mathrm{HT}}\right), \\
& \hat{\beta}=\frac{\sum_{i \in s} d_{i} q_{i} x_{i} y_{i}}{\sum_{i \in s} d_{i} q_{i} x_{i}^{2}}
\end{aligned}
$$

where

Which is equivalent to GREG estimator of $Y$ (See, Cassel et al., 1976).

An approximate variance of $\hat{Y}_{C}$ of $Y$ for a large sample is given by (Deville and Sarndal, 1992)

$V\left(\hat{Y}_{C}\right)=-\frac{1}{2} \sum_{i=1}^{N} \sum_{j=1}^{N} D_{i j}\left(d_{i} E_{i}-d_{j} E_{j}\right)^{2}$

Where $E_{i}=y_{i}-\beta x_{i}$, and $\beta=\frac{\sum_{i=1}^{N} d_{i} q_{i} x_{i} y_{i}}{\sum_{i=1}^{N} d_{i} q_{i} x_{i}^{2}}$,

An attempt has been made in the present paper to develop calibration estimator of finite population mean in two-stage stratified random sampling. Following the calibration approach of Deville and Sarndal (1992) \& calibration based estimator in two-stage sampling Aditya et al., (2016) when auxiliary information on a single auxiliary variable $x$ related to study variable $y$ is known at first stage unit (fsu) level, that means the total of $x$ i.e. $X=\sum_{i=1}^{N_{I}} x_{i}$, at $i^{\text {th }}$ fsu level is known. The usual estimator of population mean of the study variate $y$ in two-stage stratified random sampling has been developed in section- 2 .

The calibration estimators of the population mean in two-stage stratified random sampling by calibrating the design weight have been developed in the section-3. The variances and variance estimators of the calibration estimators have been developed in the section4 and a limited simulation study has been conducted in the section-5.

The usual estimator of population mean in two-stage stratified random sampling

Consider the finite population $\left(U=U_{1}, U_{2}, U_{3}, \ldots \ldots \ldots \ldots, U_{N}\right)$ consists of $N$ first stage units (fsu) and is stratified into $L$ strata such that $U_{t}$ consists of $N_{t}$ fsu and $\sum_{t=1}^{L} \mathrm{~N}_{t}=\mathrm{N}$. We also consider that each fsu in $\mathrm{t}^{\text {th }}$ stratum $(t=1,2,3, \ldots \ldots \ldots . ., L)$ has $M_{t}$ number of second stage units (ssu). Now the following terms are define as

$Y_{t i j}=$ value of the characteristic y under study on $\mathrm{j}^{\text {th }} \operatorname{ssu}\left(j=1,2,3, \ldots \ldots \ldots \ldots . . ., M_{t}\right)$ corresponding to the $\mathrm{i}^{\text {th }}$ fsu $\left(i=1,2,3, \ldots \ldots \ldots . . ., N_{t}\right)$ in the $\mathrm{t}^{\text {th }}$ stratum.

$\bar{Y}_{t i .}=\frac{1}{\mathrm{M}_{t}} \sum_{j=1}^{\mathrm{M} t} \mathrm{Y}_{t i j}=\frac{1}{\mathrm{~N}_{t}} \sum_{i=1}^{\mathrm{N} t} \overline{\mathrm{Y}}_{t i}$, mean of ssu's of $\mathrm{i}^{\text {th }}$ fsu in $\mathrm{t}^{\text {th }}$ stratum.

$\bar{Y}_{t . .}=\frac{1}{\mathrm{~N}_{t} \mathrm{M}_{t}} \sum_{i=1}^{\mathrm{N}_{t}} \sum_{j=1}^{\mathrm{M}_{t}} \mathrm{Y}_{t i j}$, the population mean of $y$ in $\mathrm{t}^{\text {th }}$ stratum. 
$\overline{\mathrm{Y}}=\frac{\sum_{t=1}^{L} \overline{\mathrm{Y}}_{t . .} \mathrm{M}_{t} \mathrm{~N}_{t}}{\sum_{t=1}^{L} \mathrm{M}_{t} \mathrm{~N}_{t}}$, the population mean of $Y$

in the population.

$$
\begin{aligned}
& S_{t i y}^{2}=\frac{1}{\left(\mathrm{M}_{t}-1\right)} \sum_{j=1}^{\mathrm{M}_{t}}\left(\mathrm{Y}_{t i j}-\overline{\mathrm{Y}}_{t i .}\right)^{2} \text { and } \\
& S_{t b y}^{2}=\frac{1}{\left(\mathrm{~N}_{t}-1\right)} \sum_{i=1}^{N_{t}}\left(\overline{\mathrm{Y}}_{t i .}-\overline{\mathrm{Y}}_{t . .}\right)^{2}
\end{aligned}
$$

Now, consider that a sample of size $n_{t}$ fsu's out of $N_{t}$ fsu's is selected from $\mathrm{t}^{\text {th }}$ stratum and sub-samples of size $m_{t}$ out of $M_{t}$ ssu's from the selected $n_{t}$ fsu's are drawn by SRSWOR (simple random sampling without replacement). This process is carried out independently in each stratum. We further define

$\bar{y}_{t i}=\frac{1}{m_{t}} \sum_{j=1}^{m_{t}} y_{t i j}$, sample mean from $\mathrm{i}^{\text {th }}$ selected fsu $\left(i=1,2,3, \ldots \ldots \ldots \ldots, n_{t}\right)$ in $\mathrm{t}^{\text {th }}$ stratum.

$\bar{y}_{t s}=\frac{1}{n_{t}} \sum_{i=1}^{n_{t}} \bar{y}_{t i}$, the overall sample mean in $\mathrm{t}^{\text {th }}$ stratum.

$s_{t i y}^{2}=\frac{1}{m_{t}-1} \sum_{j=1}^{m_{t}}\left(y_{t i j}-\bar{y}_{t i}\right)^{2}$

$s_{t b y}^{2}=\frac{1}{n_{t}-1} \sum_{i=1}^{n_{t}}\left(\bar{y}_{t i}-\bar{y}_{t s}\right)^{2}$

Obviously, the $\bar{y}_{t s}$ is an unbiased estimator of $\overline{\mathrm{Y}}_{t . .}$. The variance of $\bar{y}_{t s}$ is obtained as
$V\left(\bar{y}_{t s}\right)=\left(\frac{1}{n_{t}}-\frac{1}{\mathrm{~N}_{t}}\right) S_{t b y}^{2}+\frac{1}{n_{t}}\left(\frac{1}{m_{t}}-\frac{1}{\mathrm{M}_{t}}\right) S_{t w y}^{2}$

Where $S_{t w y}^{2}=\frac{1}{\mathrm{~N}_{t}} \sum_{i=1}^{\mathrm{N}_{t}} S_{t i y}^{2}$

An unbiased estimator of $V\left(\bar{y}_{t s}\right)$ is obtained as

$\hat{V}\left(\bar{y}_{t s}\right)=\left(\frac{1}{n_{t}}-\frac{1}{\mathrm{~N}_{t}}\right) s_{t b y}^{2}+\frac{1}{\mathrm{~N}_{t}}\left(\frac{1}{m_{t}}-\frac{1}{\mathrm{M}_{t}}\right) s_{t w y}^{2}$

Where $s_{t w y}^{2}=\frac{1}{n_{t}} \sum_{i=1}^{n_{t}} s_{t i y}^{2}$

An unbiased estimator of $\bar{Y}$ in stratified two stage random sampling is given by $\bar{y}_{s}=\sum_{t=1}^{L} W_{t} \bar{y}_{t s}(7)$

Where $W_{t}=\frac{\mathrm{N}_{t}}{\mathrm{~N}}$ such that $\sum_{t=1}^{L} W_{t}=1$

The variance of $\bar{y}_{s}$ is obtained as

$V\left(\bar{y}_{s}\right)=\sum_{t=1}^{L} W_{t}^{2} V\left(\bar{y}_{t s}\right)$

$=\sum_{t=1}^{L} W_{t}^{2}\left[\left(\frac{1}{n_{t}}-\frac{1}{\mathrm{~N}_{t}}\right) S_{t b y}^{2}+\frac{1}{n_{t}}\left(\frac{1}{m_{t}}-\frac{1}{\mathrm{M}_{t}}\right) S_{t w y}^{2}\right]$

An unbiased estimator of $V\left(\bar{y}_{s}\right)$ is obtained as

$\hat{V}\left(\bar{y}_{s}\right)=\sum_{t=1}^{L} W_{t}^{2} \hat{V}\left(\bar{y}_{t s}\right)$

$=\sum_{t=1}^{L} W_{t}^{2}\left[\left(\frac{1}{n_{t}}-\frac{1}{\mathrm{~N}}\right) s_{t b y}^{2}+\frac{1}{\mathrm{~N}_{t}}\left(\frac{1}{m_{t}}-\frac{1}{\mathrm{M}_{t}}\right) s_{t w y}^{2}\right]$ 
Proposed calibration estimators of population mean in two-stage stratified random sampling

We have described details of development of an estimator of population mean $\overline{\mathrm{Y}}=\sum_{t=1}^{L} \sum_{i=1}^{\mathrm{N}_{t}} \sum_{j=1}^{\mathrm{M}_{t}} \frac{y_{t i j}}{\mathrm{M}_{0}}, \mathrm{M}_{0}=\sum_{t=1}^{L} \mathrm{~N}_{t} \mathrm{M}_{t}$ using simple random sampling without replacement (SRSWOR) independently in each stratum.

The estimator of $\bar{Y}$ is given by

$\bar{y}_{s}=\sum_{t=1}^{L} W_{t} \bar{y}_{t s}$

Such that $\sum_{t=1}^{L} W_{t}=1$. The weight $W_{t}$ is self design weight and it is given by $\frac{\mathrm{N}_{t}}{\mathrm{~N}}$, and $\mathrm{N}=\sum_{t=1}^{L} \mathrm{~N}_{t}$.

The variance of $\bar{y}_{s}$ is obtained as

$V\left(\bar{y}_{s}\right)=\sum_{t=1}^{L} W_{t}^{2}\left[\left(\frac{1}{n_{t}}-\frac{1}{\mathrm{~N}_{t}}\right) S_{t b y}^{2}+\frac{1}{n_{t}}\left(\frac{1}{m_{t}}-\frac{1}{\mathrm{M}_{t}}\right) S_{t w y}^{2}\right]$

An unbiased estimator of $V\left(\bar{y}_{s}\right)$ is obtained as

$\hat{V}\left(\bar{y}_{s}\right)=\sum_{t=1}^{L} W_{t}^{2}\left[\left(\frac{1}{n_{t}}-\frac{1}{\mathrm{~N}}\right) s_{t b y}^{2}+\frac{1}{\mathrm{~N}_{t}}\left(\frac{1}{m_{t}}-\frac{1}{\mathrm{M}_{t}}\right) s_{t w y}^{2}\right]$

The weight $W_{t}$ can be calibrated if the information of an auxiliary variable $x$ related to the study variate $y$ is available in order to improve the efficiency of the estimator $\bar{y}_{s}$. The information of an auxiliary variable $\mathrm{x}$ related to y may be available at fsu's level in twostage stratified random sampling.
In this case, the population mean of the auxiliary variable can easily be obtained, i.e.

$\overline{\mathrm{X}}=\sum_{t=1}^{L} \sum_{i=1}^{N_{t}} \frac{X_{t i}}{\mathrm{M}_{\mathrm{O}}}$

Where $X_{t i}$ is the value of the auxiliary variable corresponding to $i^{\text {th }}$ fsu in the $t^{\text {th }}$ stratum.

Let $W_{t}^{\prime}$ be calibrated weight.The calibration estimator of $\bar{Y}$ is therefore, given by

$\bar{y}_{s c}=\sum_{t=1}^{L} W_{t}^{\prime} \bar{y}_{t s}$

Where $W_{t}^{\prime}$ is calibrated weight obtained by minimizing a distance measure $\sum_{t=1}^{L}\left(W_{t}^{\prime}-W_{t}\right)^{2} / q_{t} W_{t}$, where $q_{t}$ is positive quantity unrelated to $W_{t}$, subject to calibration constraint

$\sum_{t=1}^{L} W_{t}^{\prime} \bar{x}_{t s}=\overline{\mathrm{X}}(15)$

Where $\overline{\mathrm{X}}_{\mathrm{ts}}$ is an estimator of $\overline{\mathrm{X}}_{t}$, developed similarly as $\bar{y}_{t s}$. For the ready reference, $\bar{x}_{t s}$ is given by

$\bar{x}_{t s}=\frac{1}{n_{t}} \sum_{i=1}^{n_{t}} \bar{x}_{t i}$, Where $\bar{x}_{t i}=\frac{1}{m_{t}} \sum_{j=1}^{m_{t}} x_{t i j}$,

The following function

$\phi\left(W_{t}^{\prime}, \lambda\right)=\sum_{t=1}^{L} \frac{\left(W_{t}^{\prime}-W_{t}\right)^{2}}{q_{t} W_{t}}+2 \lambda \sum\left(W_{t}^{\prime} \bar{x}_{t s}-\bar{X}\right)$

Is minimized with respect to $W_{t}^{\prime}$, where $\lambda$ is Langrangian multiplier. This yields $W_{t}^{\prime}$ as 


$$
W_{t}^{\prime}=W_{t}\left[1+\frac{\bar{X}-\sum W_{t} \bar{x}_{t s}}{\sum W_{t} q_{t} \bar{x}_{t s}^{2}} q_{t} \bar{x}_{t s}\right]
$$

The developed calibration estimator is given by

$$
\bar{y}_{s c}=\sum_{t=1}^{L} W_{t} \bar{y}_{t s}+\frac{\sum_{t=1}^{L} W_{t} \bar{t}_{t s} \bar{y}_{t s} q_{t}}{\sum_{t=1}^{L} W_{t} \bar{x}_{t s}^{2} q_{t}}\left(\bar{X}-\sum_{t=1}^{L} W_{t} \bar{x}_{t s}\right)
$$

Which is a combined regression estimator in stratified random sampling. $\bar{y}_{s c}$ is a class of estimators depending upon the value of $q_{t}$.

For $q_{t}=1$, we get an estimator as

$$
\bar{y}_{S C 1}=\sum_{t=1}^{L} W_{t} \bar{y}_{t s}+\frac{\sum_{t=1}^{L} W_{t} \bar{x}_{t s} \bar{y}_{t s}}{\sum_{t=1}^{L} W_{t} \bar{x}_{t s}^{2}}\left(\bar{X}-\sum_{t=1}^{L} W_{t} \bar{x}_{t s}\right)
$$

Which is a combined regression estimator in two-stage stratified random sampling.

For $q_{t}=\frac{1}{\bar{x}_{t s}}$, we get another estimator as

$$
\begin{aligned}
& \bar{y}_{S C 2}=\sum_{t=1}^{L} W_{t} \bar{y}_{t s}+\frac{\sum_{t=1}^{L} W_{t} \bar{y}_{t s}}{\sum W_{t} \bar{x}_{t s}}\left(\bar{X}-\sum_{t=1}^{L} W_{t} \bar{x}_{t s}\right) \\
& =\frac{\sum_{t=1}^{L} W_{t} \bar{y}_{t s}}{\sum_{t=1}^{L} W_{t} \bar{x}_{t s}} \bar{X}(21)
\end{aligned}
$$

Which is a combined ratio estimator in twostage stratified random sampling.

\section{Variance and variance estimator of proposed calibration estimators}

The approximate variance of $\bar{y}_{\mathrm{scl}}$ has been derived following the procedure given by Sarndal et al., (2003, chapter 4\&8), and is given by

$$
V\left(\bar{y}_{s c 1}\right)=\sum_{t=1}^{L} \frac{W_{t}^{2}}{M_{t}^{2} N_{t}^{2}}\left[V_{t p s u}+V_{t s s u}\right]
$$

$V_{t p s u}=\sum_{i=1}^{N_{t}} \sum_{j=1}^{N_{t}} \frac{\Delta_{l i j} D_{i} D_{j}}{\pi_{l i} \pi_{l j}}$,

Where $\quad \Delta_{i j}=-\frac{n_{t}\left(N_{t}-n_{t}\right)}{N_{t}^{2}\left(N_{t}-1\right)}, \quad \pi_{l i}=\frac{n_{t}}{N_{t}}$, $D_{i}=\bar{Y}_{t i}-\bar{Y}_{t}-B\left(\bar{X}_{t i}-\bar{X}_{t}\right)$ and $B=\frac{\sum_{t=1}^{L} W_{t} \bar{Y}_{t} \bar{X}_{t}}{\sum_{t=1}^{L} W_{t} \bar{X}_{t}^{2}}$

$V_{t s s u}=\sum_{U} V_{i} / \pi_{l i}$

Where $V_{i}=\sum \sum_{U i} \Delta_{k l / i} y_{t i j} y_{t i j} / \pi_{j / i} \pi_{j^{\prime} / i}$,

$\Delta_{k l / i}=-\frac{m_{t}\left(M_{t}-m_{t}\right)}{M_{t}^{2}\left(M_{t}-1\right)}$ and $\pi_{j / i}=m_{t} / M_{t}$

The estimator of variance of $\bar{y}_{S C 1}$ following Sarndal et al., (2003), is obtained as

$\hat{V}\left(\bar{y}_{s c}\right)=\sum_{i=1}^{L} \frac{W_{i}^{2}}{N_{t}^{2} M_{t}^{2}}\left[\frac{N_{t}\left(N_{t}-n_{t}\right)}{n_{t}^{t}}\left\{\sum \sum_{w} \frac{\left(-d_{i} d_{j}\right)}{n_{t}-1}-\sum_{w} \hat{V}_{i}^{2}\right\}+\frac{N_{t}^{2}}{n_{t}^{2}} \sum_{i} \hat{V}_{i}^{2}\right]$

Where $\hat{V}_{i}^{2}=\frac{-\left(M_{t}-m_{t}\right) M_{t}}{m_{t}^{2}\left(m_{t}-1\right)} \sum \sum_{s i} y_{t i j} y_{t i j^{\prime}}$,

$d_{i}=\bar{y}_{t i}-\bar{y}_{t s}-\hat{B}\left(\bar{x}_{t i}-\bar{x}_{t s}\right)$,

and 
Table. 1 The estimate of $\bar{Y}$ based on $\bar{y}_{s}, \bar{y}_{s c 1}$ and $\bar{y}_{s c 2}$ along with their estimate of variance

\begin{tabular}{|c|c|c|c|c|}
\hline Estimator & Estimate & \% RB & $\begin{array}{c}\text { Estimate of } \\
\text { variance }\end{array}$ & PSE \\
\hline$\overline{\mathrm{y}}_{\mathrm{s}}$ & 26.77 & 6.92 & 13.15 & 13.54 \\
\hline$\overline{\mathrm{y}}_{\mathrm{scl}}$ & 29.12 & 1.25 & 8.05 & 9.74 \\
\hline$\overline{\mathrm{y}}_{\mathrm{sc} 2}$ & 29.16 & 1.39 & 10.75 & 11.24 \\
\hline
\end{tabular}

NB: Actual population mean of $Y=29.36$

The approximate variance of $\overline{\mathrm{y}}_{\mathrm{sc} 2}$ following Sarndal et al., (2003), and Singh et al., (1998), is obtained as

$V\left(\bar{y}_{s c 2}\right)=\left(\frac{\bar{X}}{\bar{x}_{s}}\right)^{2} \sum_{t=1}^{L} \frac{W_{t}^{2}\left(1-f_{t}\right)}{n_{t}} S_{E t}^{2}$

Where $S_{E t}^{2}=\frac{1}{N_{t}-1} \sum_{t=1}^{N_{t}} E_{t i}^{2}$

$E_{t i}=\bar{Y}_{t i}-\bar{Y}_{t}-B\left(\bar{X}_{t i}-\bar{X}_{t}\right), \quad f_{t}=n_{t} / N_{t} \quad$ and $B=\sum_{t=1}^{L} W_{t} \bar{Y}_{t i} / \sum_{t=1}^{L} W_{t} \bar{X}_{t i}$.

The approximate consistent and unbiased estimator of $\mathrm{V}\left(\overline{\mathrm{y}}_{\mathrm{sc} 2}\right)$ is obtained as $\hat{V}\left(\bar{y}_{s c 2}\right)=\left(\frac{\bar{X}}{\bar{x}_{s}}\right)^{2} \sum_{t=1}^{L} \frac{W_{t}^{2}\left(1-f_{t}\right)}{n_{t}} s_{e t}^{2}$

Where $\mathrm{s}_{\mathrm{et}}^{2}=\frac{1}{\mathrm{n}_{\mathrm{t}}-1} \sum_{\mathrm{i}=1}^{\mathrm{n}_{\mathrm{t}}} \mathrm{e}_{\mathrm{ti}}^{2}$

$e_{t i}=\bar{y}_{t i}-\bar{y}_{t s}-B\left(\bar{x}_{t i}-\bar{x}_{t s}\right)$,

$\hat{B}=\sum_{t=1}^{L} W_{t} \bar{y}_{t s} / \sum_{t=1}^{L} W_{t} \bar{x}_{t s}$, and $\bar{x}_{s}=\sum_{t=1}^{L} W_{t} \bar{x}_{t s}$ is

an estimate of $\bar{X}$ in two-stage stratified random sampling.

\section{Simulation}

A limited simulation study has been carried out with real data. The population MU284 given in Appendix-C of Sarndal et al., (2003) has been used. There are 50 fsu's of varying size. The variable under study $(y)_{\text {is }}$ population of 1985 and an auxiliary variable $(x)$ is the population of 1975. The 50 fsu's are stratified into 4 strata considering the value of $x$ in ascending order. The stratum $\mathrm{I}$ consists of 13 fsu's, stratum II consists of 14 fsu's, stratum III consists of 12 fsu's, stratum IV consists of 11 fsu's respectively.

The samples of size 4 fsu's were drawn by SRSWOR independently from strata 1 to 4 , respectively. This process has been repeated 300 times independently. That means, we obtained 300 samples of size 4 fsu's from each stratum. Sub samples of size 3 ssu's are drawn by SRSWOR from each sample of fsu's in each stratum. The values of $y$ and $x$ in sub samples were used to compute the population mean. In this process, we get 300 estimates of $\bar{Y}_{t}$ from 300 sub samples in each stratum.

The values of $\mathrm{y}$ and $\mathrm{x}$ in sub samples were used to compute the population mean of $\bar{Y}$.

In this process, we get 300 estimates of $\bar{Y}_{t}$ from 300 sub-samples in each stratum. The averages of these 300 estimates from each stratum are used to get the estimate of $\bar{Y}$. Mathematically, let $\hat{\theta}_{t i}$ be the estimate of $\bar{Y}_{t}$ from $i^{\text {th }}$ stratum. We compute 
$\hat{\theta}_{t}=\frac{1}{300} \sum_{t=1}^{300} \hat{\theta}_{t i}$

The average of 300 sub-samples in the $\mathrm{t}^{\text {th }}$ stratum. So, we get simulated estimate of $\bar{Y}$ as follows

$\hat{\theta}=\sum_{t=1}^{L} W_{t} \hat{\theta}_{t}$

The percent relative bias $(\% \mathrm{RB})$ of the estimate has been computed as follows

$$
\% R B=\frac{|\hat{\theta}-\theta|}{\theta} \times 100
$$

Similarly, the approximate variances of the usual estimator $\overline{\mathrm{y}}_{\mathrm{s}}, \quad \overline{\mathrm{y}}_{\mathrm{sc1}}$ and $\overline{\mathrm{y}}_{\mathrm{sc} 2}$ are computed. The percent standard error (PSE) of the estimate has been computed as follows:

$$
P S E=\frac{S E(\hat{\theta})}{\hat{\theta}} \times 100
$$

The simulation studies results are presented in the Table 1.

It has been found from the results of the Table 1 that the regression type calibration estimator $\overline{\mathrm{y}}_{\mathrm{sc1} 1}$ and ratio type calibration estimator $\overline{\mathrm{y}}_{\mathrm{sc} 2}$ have performed better than the usual estimator $\bar{y}_{s}$ in two-stage stratified random sampling. However, estimator $\overline{\mathrm{y}}_{\mathrm{scl}}$ has been found best in comparison to estimator $\overline{\mathrm{y}}_{\mathrm{sc} 2}$ as it has minimum PSE of 9.74 as against 11.24 for $\bar{y}_{\text {sc2 }}$. It may be noted that the calibration estimator $\overline{\mathrm{y}}_{\mathrm{scl}}$ is equivalent to combined weighted regression estimator and $\bar{y}_{\mathrm{sc} 2}$ is the usual combined ratio estimator. It has also been found that the calibration estimator $\overline{\mathrm{y}}_{\mathrm{scl}}$ is relatively less biased than estimators $\overline{\mathrm{y}}_{\mathrm{sc} 2}$ and $\overline{\mathrm{y}}_{\mathrm{s}}$

\section{References}

Aditya, K., Sud, U.C., Chandra, H. and Biswas, A. 2016. Calibration based regression type estimator of the population total under two-stage sampling design. Journal of Indian Society of Agricultural Statistics, Vol. 70(1), pp. 19-24.

Cassel, C.M., Sarndal, C.E. and Wretman, J.H. 1976. Some results on generalized difference estimation and generalized regression estimation for finite population, Biometrika, Vol. 63, pp. 615-620.

Deville, J.C. and Sarndal, C.E. 1992. Calibration estimators in survey sampling. Journal of the American Statistical Association, Vol. 87, pp. 376-382.

Horvitz, D.G. and Thompson, D.J. 1952. A generalization of sampling without replacement from a finite universe. Journal of the American Statistical Association, Vol. 47, pp. 663-685.

Kim, J.K. and Park, M. 2010. Calibration estimation in survey sampling. International Statistical Review, Vol. 78, 21-39.

Mourya, K.K., Sisodia, B.V.S. and Chandra, H. 2016. Calibration approach for estimating finite population parameter in two-stage sampling. Journal of Statistical Theory and Practice, Vol. 10(3), pp. 550-562.

Sarndal, C.E., Swensson, B. and Wretman, J. 2003. Model-assisted survey sampling. Springer-Verlag, New York, Inc. (Revised Edition).

Singh, S., Horn, S. and Yu, F. 1998. Estimation of variance of the general 
regression estimators: Higher level calibration approach. Survey methodology, Vol. 24(1), pp. 41-50.

Sinha, N., Sisodia, B.V.S., Singh, S. and Singh, S. K. 2016. Calibration approach estimation of mean in stratified sampling and double stratified sampling. Communication in StatisticsTheory and Methods, 46(10), pp. 49324942.
Sud, U.C., Chandra, H. and Gupta, V.K. 2014. Calibration based product estimator in single and two phase sampling. Journal of Statistical Theory and Practice, 8, pp. 1-11.

Tracy, D.S., Singh, S. and Arnab, R. 2003. Note on calibration estimators in stratified and double sampling. Survey Methodology, 29, pp. 99-106.

\section{How to cite this article:}

Sandeep Kumar. 2018. Calibration Approach Based Estimators of Finite Population Mean in Two - Stage Stratified Random Sampling. Int.J.Curr.Microbiol.App.Sci. 7(01): 1808-1815. doi: https://doi.org/10.20546/ijcmas.2018.701.219 